\title{
Phenome-Wide Association Studies: Embracing Complexity for Discovery
}

\author{
Sarah A. Pendergrass ${ }^{a}$ Anurag Verma ${ }^{c}$ Anna Okula ${ }^{c}$ Molly A. Hall ${ }^{c}$ \\ Dana C. Crawford ${ }^{\mathrm{b}}$ Marylyn D. Ritchie ${ }^{\mathrm{a}, \mathrm{c}}$ \\ ${ }^{a}$ Biomedical and Translational Informatics Program, Geisinger Health System, Danville, Pa., USA, ${ }^{b}$ Department of \\ Epidemiology and Biostatistics, Institute for Computational Biology, Case Western Reserve University, Cleveland, \\ Ohio, and ${ }^{\mathrm{C} C e n t e r}$ for Systems Genomics, Pennsylvania State University, University Park, Pa.,USA
}

\section{Key Words}

Genome-wide association studies · Phenome-wide association studies $\cdot$ Phenomics

\begin{abstract}
The inherent complexity of biological systems can be leveraged for a greater understanding of the impact of genetic architecture on outcomes, traits, and pharmacological response. The genome-wide association study (GWAS) approach has well-developed methods and relatively straight-forward methodologies; however, the bigger picture of the impact of genetic architecture on phenotypic outcome still remains to be elucidated even with an evergrowing number of GWAS performed. Greater consideration of the complexity of biological processes, using more data from the phenome, exposome, and diverse-omic resources, including considering the interplay of pleiotropy and genetic interactions, may provide additional leverage for making the most of the incredible wealth of information available for study. Here, we describe how incorporating greater complexity into analyses through the use of additional phenotypic data and widespread deployment of phenome-wide association studies may provide new insights into genetic factors influencing diseases, traits, and pharmacological response.

(c) 2015 S. Karger AG, Basel
\end{abstract}

\section{Introduction}

Biological systems are incredibly complex, with networks and feedback existing between genetic architecture, signaling pathways, intermediate phenotypes, and outcome traits. This complexity is both spatial and temporal in nature, from the three-dimensional structure of DNA in the nucleus [1], to the regulation of transcription and translation [2], to cell type specificity and the location of cells, and the effect of environmental exposure and age [3]. Thus, the architecture underlying human diseases (such as hypertension, asthma, etc.) as well as the response to drugs (antihypertensives for high blood pressure, albuterol for asthma, etc.) can vary from individual to individual. A single disease, disease outcome, trait, or drug response can be the direct result of genetic variation as well as variability in how the network and feedback has been affected by interaction with other genetic variation and the environment.

Accounting for this inherent complexity when trying to uncover the etiology of a disease and drug response is a key challenge for the future of genetic epidemiology and pharmacogenomics. Much of the focus in genetic epidemiology has been on the association between single genetic variants and single outcomes, often dichotomized into 'cases' and 'controls'. A limited number of strong as-

\section{KARGER 125}

(c) 2015 S. Karger AG, Base

0001-5652/15/0794-0111\$39.50/0 
sociations have been found, leading to the discussion of which directions research should be taking in the future to better understand how genetic variation affects a disease and drug response [4]. One way to elucidate additional information about the impact of genetic architecture on outcome is to incorporate more of the complexity underlying complex traits, and leveraging this complexity in novel ways to highlight critical contributors to diseases and drug response.

Methodologies that show promise for leveraging this complexity include using phenomics approaches. Through using more phenotypic data, from disease information to subphenotypes and an array of other measures, patients with a disease can be subset based on a wide array of detailed clinical laboratory variables and other criteria, beyond just the 'presence/absence' of the disease (e.g. type 2 diabetes) or one type of quantitative measure [e.g. low-density lipoprotein cholesterol (LDL-C) levels]. Comprehensive phenotypic data can also be used in phenome-wide association studies (PheWAS) to identify biologically relevant associations and potential pleiotropy that can highlight important connections between networks of phenotypes and genetic variation.

On the genomic level, additional complexity that can be incorporated into analyses includes considering lower-frequency genetic variants along with common single nucleotide polymorphisms (SNPs), requiring new analytic methods beyond linear regression and additive encoding models. Furthermore, with the complex nature of the networks that exist in biological systems, genetic models that incorporate multiple SNPs or genes may more accurately reflect and predict the underlying biology behind phenotypic variation. Models that detect epistasis, where interactions between genes or SNPs result in changes in phenotype, could prove important for novel discoveries [5]. Thus, genetic interactions can be considered, using more complex models of SNP-SNP (SNP $\times$ $S N P)$ and gene-gene interactions $(G \times G)$. In addition, other forms of -omic data can be brought to these complex predictive models, from copy number variation to gene expression and methylation data [6]. These more complex genetic models can also be used in conjunction with PheWAS to explore the connection between phenome and genome at an unprecedented level.

Finally, the environment plays a key role in how genetic variation contributes to phenotypic variation from individual to individual, and these complex data should also be included in analyses. Collecting comprehensive environmental data can be a challenge, from choosing which environmental variables to collect to the feasibility of collecting multiple environmental exposure measures over thousands of individuals. Headway is being made in collecting more high-throughput environmental exposure data, and environmental exposure data are now being collected at an unprecedented rate. Examples include the use of PhenX variables, such as those being collected by the Marshfield Personalized Medicine Project (Marshfield PMRP) in order to link exposures and harmonized measures to electronic health record (EHR) data [7-12]. Another example is the expanding collection of environmental exposures, such as monitoring activity levels using geographic information systems (GIS), global positioning system (GPS) technology, and accelerometers [13-15]. The incorporation of these data into predictive models of the association between genetic variation and outcome may provide the 'missing pieces' that augment genotype when explaining phenotype.

\section{Phenomics}

Phenomics has been championing the collection of more phenotypic data at multiple levels of granularity [16-18]. While the entire phenome can never be truly measured, technologies for the high-throughput collection of many phenotypes are becoming more available. In model organisms, tools such as phenotype microarrays for microbial cells have now been developed that evaluate nearly 2,000 phenotypes of a microbial cell in a single experiment [19]. In humans, there are a nearly endless number of ways to measure phenotypic variation and, in turn, multiple levels at which to measure the relationship between genotype and phenotype, as the phenome varies in every cell from minute to minute. From gene expression to clinical laboratory variables (e.g. white blood cell counts and glucose measures) to diagnoses (e.g. hypertension), different details of biological processes can be measured. Examples of rich collections of phenotypic data in humans include the National Health and Nutrition Examination Surveys (NHANES), conducted by the Centers for Disease Control and Prevention (CDC; http://www.cdc.gov/nchs/nhanes.htm). Another example are the measures available within EHR, where patients have collections of International Classification of Disease (ICD) billing codes, free text, longitudinal clinical laboratory variable measures, and imaging data. To date, ICD-9 codes and phenotyping algorithms have both shown utility in association studies [20-23].

EHR data provide a unique opportunity to follow clinical data trends in a longitudinal manner. Phenotypic 
dense surveys, such as NHANES, are cross-sectional and lack longitudinal data altogether. There are several epidemiological cohorts that systematically collect longitudinal phenotypic data [24-35]. However, what sets EHR data apart from these traditional epidemiological cohorts is the fact that the longitudinal data are collected in a clinical setting, complete with comorbidities and treatments.

Phenotypic outcomes are a feature of time, environment, and genetic architecture. Diagnoses, the severity of a condition or diagnosis, clinical laboratory variables, and medication usage change over the lifetime of an individual, depending on acute scenarios (e.g. influenza) and conditions that are common with increasing age (e.g. cataracts, hypertension). Further research could be directed to better mining of both medication and comorbidity data from EHR by extending existing approaches, such as recent work mining large adverse event-reporting systems [36]. The medication data within EHR could prove incredibly useful for pharmacogenomic studies, as many drug studies are small in sample size and do not incorporate the effects of drug interactions. Because EHRs have large numbers of individuals, exposed to multiple drugs across their life course, some of those drugs taken at the same time, important relationships between genetic variation and pharmacological response as well as the factor of time and age could be highlighted.

With advancements in automating the extraction of clinical laboratory variables as well as the use of free text and medication information in a longitudinal manner, clinical data can provide a way to identify important indicators and precursors of risk for disease. There are dedicated and robust methods for longitudinal data analysis from biostatistics such as generalized linear and mixed effect models that can be applied to these data [37]; however, the adoption of these methods in genetic association studies has been slow [38]. More efforts are needed not just in the data extraction process, as described above, but also in the proper and more powerful analysis made possible by these longitudinal clinical data contained within the EHR.

The rich complexity of a wide range of measures from comprehensive collections of phenotypic data can be used in more integrated ways to better subset patients based on the totality of their health information [16]. A disease diagnosis is made up of many criteria that an individual may or may not possess, with the underlying symptoms ranging from mild to severe. Some diseases have a more heterogeneous presentation of underlying symptoms than others, such as the wide range of symptoms and severity for autism (now classified as autism spectrum disorder - a spectrum rather than one disease). Even Mendelian diseases, which are classically considered simple genetic disorders, range in presentation and severity, such as cystic fibrosis [39]. With the complexity of the networks of biological systems, the likelihood that every person with the same disease has exactly the same underlying genetic architecture is unlikely, and comprehensive phenotypic data can be leveraged to overcome this genetic heterogeneity. Subsetting patients based on phenotype has been used successfully in gene expression research, where microarray data collected across many individuals and thousands of genes can be considered high-throughput phenotypic data. For example, the clustering of these gene expression data and the use of heat maps have identified key gene expression signatures for breast cancer subtypes [40] and potential subgroupings of patients with the autoimmune disorder systemic sclerosis [41-43]. Clustering has also been performed to subset patients with asthma by a series of clinical measures to identify subgroups with differences in clinical response to treatment [44] and to reveal the multi-dimensionality of phenotypes comprising asthma and chronic obstructive pulmonary disease [45]. In a different example, 70 phenotypic observations per patient were collected for a study of neonatal disorder with multiple phenotypic features. Hierarchical cluster analyses indicated that the neonatal disorder under study was likely a novel disorder when compared to six related congenital syndromes, and the data suggested a novel molecular mechanism $[16,46]$.

Multiple phenotypic measures can be used to better subset individuals with a disease, and these subsets can then be used in turn for association analyses to identify common and different genetic variation contributing to disease across different subgroups. Subsetting individuals has an impact on sample size and can thus affect the power to detect significant associations. However, creating a homogeneous group of individuals with more consistent underlying genetics affecting their phenotypic data could uncover a signal with important biological insights and identify useful biomarkers lost through having a large but heterogeneous group of individuals with a wide range of genetic architecture.

There are many approaches for clustering data and/or identifying key trends across high-dimensional data that can be used to cluster patients with similar overall patterns of phenotypic variation across multiple phenotypes with methods incorporating genotypic data in addition to multiple phenotypes that are potentially correlated [47-50]. The methods employed can be supervised or unsupervised, and these approaches have been very successful in 
the analysis of gene expression data, a type of highthroughput comprehensive phenotypic measurements $[51,52]$. These methods can incorporate a known outcome or diagnosis to stratify patients before clustering (such as choosing to evaluate only individuals that have a diagnosis of chronic obstructive pulmonary disease [53]), or they can first cluster and then evaluate individuals based on similar data features without stratifying based on specific diagnostic criteria. In supervised learning, data can be clustered for individuals under study to identify key variables related to a specific diagnosis, outcome, or set of phenotypes of interest. These discovered key variables can then be tested in a new dataset to evaluate their predictive quality. Unsupervised clustering uses the data for direct clustering, within and across individuals, to determine patterns of similarity and dissimilarity. For example, the recent unsupervised clustering of patients using ICD-10 billing codes followed by the exploration of comorbidities resulted in a more complete profile for individuals than focusing on any single diagnosis [54]. In a second example, unsupervised cluster analysis was used to identify 5 asthma subphenotypes by selecting a mixture of inflammatory cellular measures and clinical variables [55].

Another important and promising approach for integrating a wide range of phenotypic data for identifying important subsets of individuals based on multiple phenotypes coupled with genotypic information is the use of networks [56]. For example, through building a network of disease genes linked to known disorders, a bipartite 'Human Disease Network' was defined that can be explored in the context of genes connected to each other via diseases, or diseases connected via genes $[57,58]$. Networks can identify highly connected clusters of data and distinct network patterns, and these network structures across subsets of individuals can be compared to identify network features that may be important $[59,60]$.

\section{Pleiotropy}

Pleiotropy has many definitions [61-63] and is often defined as one gene affecting more than one unrelated phenotype. Given that non-protein-coding elements can also play a role in pleiotropy, the definition 'one locus affecting more than one unrelated phenotype' can be a more useful description of pleiotropy. Pleiotropy may also be defined as the effect of a single SNP or genetic variant on multiple phenotypes [62].

Just as there are many definitions of pleiotropy, there are many challenges to identifying it. For example, there are many ways in which gene products can affect more than one phenotypic measure, as the route from genetic level variation to phenotypic variation is complex [64]. Determining 'unrelated' phenotypes can be highly subjective, as there are many ways in which multiple phenotypes can be interrelated and correlated. Phenotypes can be measured at different levels, such as gene expression, clinical laboratory variables, and disease outcomes, and these different levels each put investigations of pleiotropy into different contexts, making it challenging to compare pleiotropy between these levels. In the case of a single SNP that is associated with more than one phenotype, considerable follow-up investigation is required to establish if a statistical association between one SNP and multiple phenotypes is 'true' pleiotropy [62]. Even with these challenges, identifying patterns of genetic variation associated with multiple phenotypes can provide important clues about the complex networks between genetic and phenotypic variation.

There are many examples of pleiotropy in humans, such as the association of $A P O E$ with both Alzheimer's disease [65] and LDL-C [66]. The comparison of results across multiple genome-wide association studies (GWAS) has highlighted potential pleiotropy as well as potential interrelationships between phenotypes not identified through the 'one phenotype at a time' approach of GWAS. GWAS have identified pleiotropy across immune-related diseases [67-69], pancreatic cancer [70], and anthropometric [71], hematologic [72], and inflammation [73] traits. An analysis of the National Human Genome Research Institute (NHGRI) GWAS catalog [74] suggested that $\sim 17 \%$ of all genes in the catalog could be defined as pleiotropic [75]. Pleiotropy is also relevant to less common Mendelian diseases such as cystic fibrosis and may underlie the wide range of expression and symptom severity in these diseases [76].

The mapping or elucidation of human pleiotropy is important not just for understanding the biological complexity of phenotypes but also in the context of clinical care. For a hypothetical example: what if a genetic variant is not only associated with a decrease in LDL-C levels but also with an increase in hypertension risk, sometimes called 'antagonistic pleiotropy'? What if this SNP also has evidence of having a regulatory effect on the gene expression of several genes, as identified through the databases such as SCAN [77] which provides comprehensive 1000 Genomes-based eQTL measurements? Do any of these genes then have a known role in lipid or hypertension pathways? Is it possible that a drug that was directed at one of these pathways for hypertension might have an 
adverse effect on LDL-C levels? Is there a previously undiscovered biological reason for the inverse relationship found for these two phenotypes? These paths for further clinically relevant discovery would not be available when just exploring the association between genetic variation and a single phenotype.

\section{Genetic Variation: SNPs to Copy Number Variants, Epistasis and Beyond}

Thus far, this review has focused on the relevance of using far more phenotypic data for analysis than traditionally considered. Another important piece of the puzzle in identifying the impact of genetic architecture on outcome is incorporating additional genetic variation information beyond common variants. This includes using low-frequency genetic variants (minor allele frequency $<0.01$ ), as well as moving beyond single nucleotide variation to copy number variants (CNVs). Multiple challenges arise when using these other data. For low-frequency variant analyses, approaches for collapsing or binning variants for subsequent burden and/or dispersion statistical analyses become important for identifying the enrichment of lowfrequency variants among individuals with a particular disease [78-80]. Copy number variation, such as duplications and deletions, occurs at appreciable frequency across the genome [81]. CNVs have been associated with neuropsychiatric disorders such as autism and schizophrenia $[82,83]$; as such, large and rare copy number variation events [83] as well as the accumulation or copy number burden [84] are appreciated as potential contributors to disease risk. Analysis strategies beyond common variants continue to emerge as genotyping, sequencing, and copy number identification methods are refined.

Another important area of research is exploring genegene interactions. With the complex pathways, biological cascades, and feedback mechanisms that exist in biological systems, the interaction between genetic factors can also be an important contributor to phenotypic variability. Epistasis is described statistically as the departure from additivity and phenotypically as the suppression of one gene or allele's phenotype by another gene or allele $[5,85]$. It is infeasible to perform exhaustive tests for epistasis in a typical GWAS dataset. Therefore, several studies have adopted prioritization strategies to investigate genegene interactions $(G \times G)$ through evaluating the association between specific SNP $\times$ SNP regression models and phenotypic outcome. For example, several studies including studies of cataracts [11], HDL cholesterol [86], and multiple sclerosis [87] have identified SNP $\times$ SNP interactions using a Biofilter $[88,89]$ to biologically filter SNPs based on pathway information. All three studies found intriguing relationships between SNP interactions and phenotypes, highlighting potential interactions between gene products while also providing biological context for the interactions behind the identified associations. In a separate study, bipartite networks were used to build a human phenotype network within a glaucoma dataset in which epistatic interactions among prioritized SNPs were identified using a statistical epistasis network [90].

Tools exist for developing and evaluating SNP-SNP models beyond standard regression-based approaches, such as Multifactor Dimensionality Reduction (MDR) $[91,92]$ and the Analysis Tool for Heritable and Environmental Network Associations (ATHENA), and now Bayesian approaches [93-99]. Advances in data reduction methods and inclusion of other -omic data beyond SNPs, such as CNVs, as well as noncoding variants in the context of prioritization strategies may prove valuable in identifying additional epistatic relationships relevant to human phenotypes [6].

Finally, analyses incorporating epistasis, pleiotropy, and multiple phenotypes can also be explored. The tool Combined Analysis of Pleiotropy and Epistasis (CAPE) combines SNP data with multiple phenotypes and infers a directed genetic interaction network that describes how variants interact to influence a panel of phenotypes [100]. Thus far, CAPE has been used to detect and interpret epistasis in model organisms, specifically yeast, Drosophila, and mice, but can also be applied to human data [101, 102].

Beyond interactions for genetic variation data, metadimensional approaches are now also being used to combine multiple-omic measures for predictive model development [103], such as combining measures of SNPs and methylome and other -omic data for discovery [6].

\section{Bringing It Together: PheWAS}

By exploring the phenome and genome together, there is potential for understanding more of the impact of genetic architecture on outcome. Thus far, many of the examples of the use of phenomic and genetic data described here can be applied to PheWAS. PheWAS began with exploring the association between SNPs and up to thousands of phenotypes, using high-throughput linear or logistic regression depending on the phenotypes [104, 105]. These studies using SNPs for PheWAS have been pursued with 
a range of data sets, such as data from a series of epidemiological studies across genetic ancestry from the Population Architecture Using Genomics and Epidemiology (PAGE) I Study [106] as well as with data from the NHANES conducted by the CDC accessed by the Epidemiologic Architecture for Genes Linked to Environment (EAGLE) [107]. These PheWAS analyses included survey, laboratory, and medical data coupled with genetic data for exploring associations. De-identified EHR data coupled with genetic data has also been used for PheWAS. For example, through the electronic MEdical Records and GEnomics (eMERGE) network [21], EHR-based ICD-9 billing codes have been used to establish the case-control status for identifying significant associations between medical record diagnoses and genetic data [108-111]. EHR-based data coupled with SNPs selected for potential involvement in the immune and autoimmune response have also been used for PheWAS work across datasets from BioVU and the Geisinger Health System (GHS) MyCode biorepository [112]. A pilot study of 27 baseline laboratory measures from 2,547 individuals of mixed genetic ancestry from the ACTG network has been undertaken as the first clinical trial-based PheWAS to date [113].

Each of these studies has shown the ability to replicate previously reported associations, clearly demonstrating that the high-throughput PheWAS approach is feasible. In addition, these studies have also identified hypothesisgenerating novel associations. Several of these studies have investigated associations across diverse genetic ancestries $[106,107,114]$. This is important, as much of GWAS has been pursued in individuals of European ancestry, and PheWAS provides a high-throughput way to contrast association results across populations in addition to identifying novel associations in understudied populations such as African-Americans. Pleiotropy has been highlighted in some of the studies in which SNPs were associated with multiple phenotypes $[106,107,114]$ and replicated in independent datasets [106, 107, 112, 113]. The PheWAS approach continues to develop, in part because the number of datasets that can be used for a PheWAS continues to grow as the amount of highthroughput genotypic and phenotypic data increases. For humans, data repositories such as the database of Genotypes and Phenotypes (dbGaP) [115] coupled with worldwide collaborations of datasets independent of $\mathrm{dbGaP}$ make PheWAS possible for both pooled analysis as well as meta-analysis settings, the latter of which is commonly used in standard GWAS [116]. Outside of human datasets, PheWAS can be used with animal breeding research studies that collect comprehensive phenotypic data.
PheWAS can be pursued with genetic variation beyond SNPs. For example, PheWAS can be performed using low-frequency variants by using biological knowledge-driven rare-variant binning and analysis approaches, such as Biobin $[78,117]$, in conjunction with various burden tests [118] and dispersion tests, such as SKAT [79]. Thus, whole-exome chip and whole-exome sequencing data can be used with PheWAS for understanding the contribution of rare genetic variation to phenotypic outcomes. Data types beyond common and rare single nucleotide variants can also be used for PheWAS. Pilot explorations of mitochondrial variants have been performed in PheWAS [119] as well as PheWAS using admixture mapping and local ancestry calculations [120] and PheWAS using CNVs. PheWAS can even be pursued with $\mathrm{G} \times \mathrm{G}$ models derived using the methods previously described herein.

The use of phenotypic data within PheWAS can be expanded to use longitudinal measures, such as the clinical measures collected within EHR over time, for discovery. PheWAS can also be performed using clinical laboratory variables instead of genetic variation for biomarker discovery [121]. The multiple phenotypes of PheWAS can be combined through principal components analysis [47] or approaches like Lasso [122] and other methods [123] to group phenotypes before PheWAS. This can reduce multiple hypothesis testing and provide relevant grouping of phenotypes based on the underlying data. Finally, preceding PheWAS, patients can be subset by phenotypic data as described earlier in this paper.

When calculating comprehensive SNP-phenotype associations in PheWAS, the number of results from a PheWAS study can be on the order of thousands to millions, depending on the number of SNPs and phenotypes. The number of associations increases if the data are stratified by genetic ancestry, or if associations are calculated with phenotypic variables transformed and untransformed [106]. One way these results can be perused is on a result-by-result basis after applying a significance threshold, with the most significant results inspected first. Information about each of the most significant results can be explored further through comparing results to the NHGRI GWAS catalog [74] or other online sources of useful annotation. However, beyond sorting the data in this fashion, inspection of results in a high-throughput way for deeper insight becomes considerably more challenging.

Visually exploring PheWAS results is extremely useful for identifying trends in the data, and there are several software packages that provide a way to plot PheWAS results: Synthesis-View [124, 125], PheWAS-View [126], 


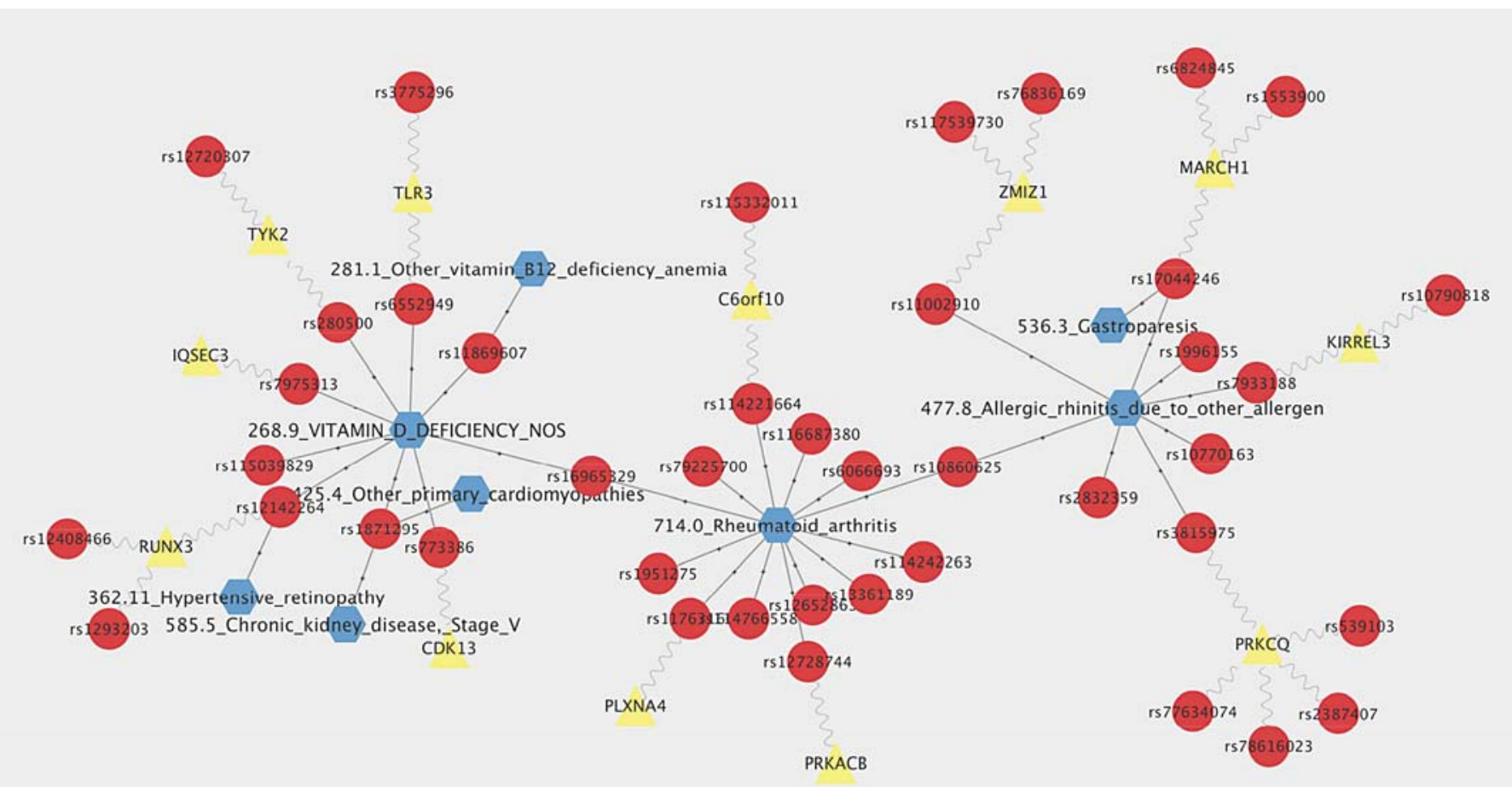

Fig. 1. Exploring a PheWAS network with Cytoscape. For a series of PheWAS results based on ICD-9 code case/control status, SNPs were first annotated with the genes they were within. Next, the genes were annotated with information connecting the genes, such as shared pathways and protein families. Finally, using Cytoscape, associated phenotypes were connected to SNPs, SNPs were con- nected to genes, and genes were connected via the shared preestablished links. After connecting SNPs (circles), genes (triangles), and phenotypes (hexagons), we created a subnetwork by selecting four levels of neighbors from the node ' 714.0 Rheumatoid Arthritis'. SNP-phenotype connections are represented by straight lines and SNP-gene connections by zigzag lines. and PhenoGram [127], in addition to other software such as R [128] and GGplot2 [129]. However, data still need to be organized and filtered before using any of these software tools for plotting results, again most powerfully facilitated through a database approach or the use of automated scripts. Exploring the co-occurrence of phenotypic outcomes and genetic variation with additional biological information visualized as a network, such as the previously described 'Human Disease Network', may better represent the genetic architecture of complex traits. For example, we have used the software tool Biofilter [88, 89 ] to annotate PheWAS results with biological knowledge, including information about genes PheWAS SNPs map within as well as known gene-gene relationships, such as pathway information from the Kyoto Encyclopedia of Genes (KEGG) [130]. Then, comprehensive PheWAS association results can be visualized as a network connecting SNPs with their associated phenotypes, using association $\mathrm{p}$ values as the edge weights where distance indicates the significance of associations using
Cytoscape [131, 132]. Cytoscape is a software package for visualizing interaction networks (http://www.cytoscape. org/). We have piloted the use of Cytoscape 3.0 to visualize significant PheWAS results and have created example networks [107] (fig. 1, 2).

Pathway-based approaches for GWAS can also be applied to PheWAS results [133], such as Pathway Analysis by Randomization Incorporating Structure (PARIS) [134]. Through the use of pathway-based approaches, information about relationships between genes and pathways can be incorporated with the results of association testing to identify key genes and biological mechanisms associated with outcome. Pathway enrichment methods could be used with the results of PheWAS to identify common involved pathways across multiple outcomes and phenotypic measures, highlighting critical pathways for drug development.

PheWAS incurs a multiple hypothesis burden greater than that of GWAS. A Bonferroni correction is a conservative approach applied to GWAS to adjust $p$ values by 


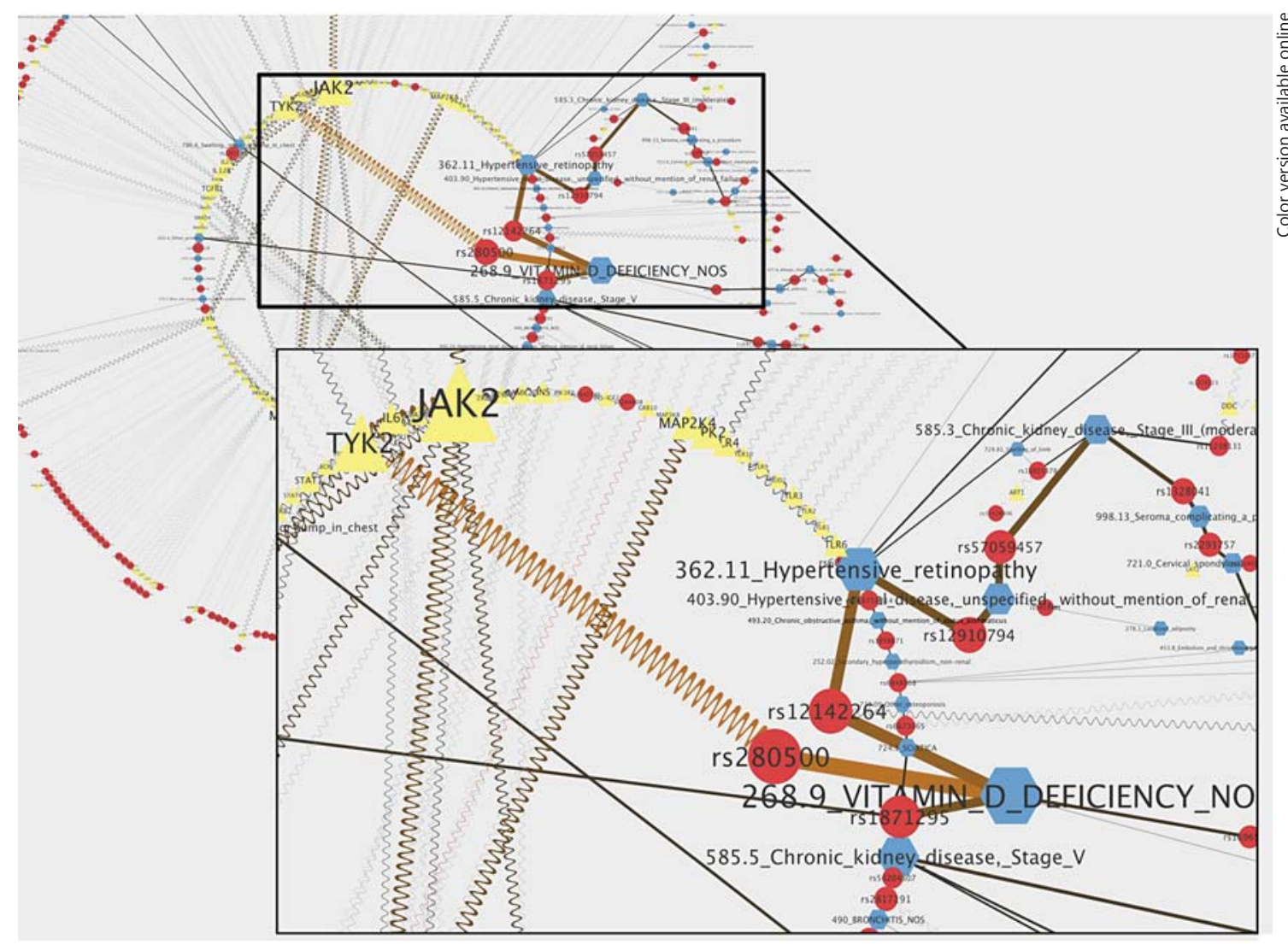

Fig. 2. Subset of a PheWAS-based network. Here, a subset of a network based on the PheWAS results of figure 1 is shown (background) after clustering the network data in a different way. Part of the image in the upper part of the plot within a box is shown in a closer view in the lower part of the plot. Bold lines indicate a more significant association between SNP and phenotype (ICD-9 code-based case/control status). Again, SNPs are represented by circles, genes by triangles, and phenotypes by hexagons. SNP-phenotype connections are represented by straight lines and SNP-gene connections by zigzag lines.

the number of tests; however, this approach assumes independence of the statistical tests. Within PheWAS, there are correlated phenotypes, and potentially correlated SNPs due to linkage disequilibrium. Other problems with current methodology for multiple hypothesis testing in GWAS have been discussed elsewhere [135]. Several PheWAS to date have used more than one dataset to seek replication of results, or have split one dataset into two smaller datasets to identify associations less likely due to chance alone or uncontrolled biases. With the ever-growing number of existing datasets in public repositories, replication in an independent dataset will become an increasingly viable option. In the future, permutation testing may become valuable for evaluating the results of PheWAS through developing an empirically derived null distribution for comparison to the unpermuted results of PheWAS to establish a significance threshold for results.
One potential risk with permutation testing is potentially an overconservative threshold for significance. More exploratory work with permutation testing and PheWAS will help develop a clearer picture of the promise and limitations for this approach specific to different types of data used in PheWAS.

\section{Environment and Exposome}

Variation in environmental exposure and variation in genetic architecture are known contributors to many complex traits and diseases. However, the identification of susceptibility genes and environmental variables that predict outcomes for complex traits and disease, as well as the characterization of the complex interplay between the two, has been understudied. The paradigm for the 
past 20 years has been to only analyze single genetic variant effects primarily through GWAS. These studies have been largely pursued without incorporating environmental exposure information, and this has hindered the ability to identify both novel genetic effects associated with disease through their interactions with the environment and may be a contributor to the identification mostly of main-effect variants of the small effect of existing GWAS results [74].

For example, in autism, there are likely varying contributions from genetic variation and the environment. There are demographic factors that appear to be related to autism, including advanced parental age and short inter-pregnancy interval [136-138], medical factors, such as drug exposure and prenatal vitamin use (particularly folate consumption levels), that have been associated with autism [139-145]. There is also a growing body of evidence of environmental risk factors, including flame retardants, pesticides, and air pollutants [146-148]. For instance, the impact of exposure to traffic-related air pollution on prenatal development and the risk of autism spectrum disorders have recently been explored in several studies [149-154]. Studies like this demonstrate the importance of considering the environment in the study of complex traits.

The measurement, including the timing of measurement, of environmental exposures is notoriously difficult, and a description of current strategies is beyond the scope of this review. However, it is worth noting that overall, there are growing contemporary attempts to collect meaningful environmental exposure data that can be used for the study of gene-environment interactions $(G \times E)$ such as air quality measures across the globe. There is also increasing use of GIS in studies. GIS are automated systems for capturing and analyzing data connected to respective geographically referenced information. Studies are also incorporating digital means to collect environmental data including GPS devices and accelerometers worn by participants. An example of comprehensive exposure data being collected for study that is linked to health data, and in the future genetic data, is the Marcellus Shale Initiative [155]. The goal of this project is to identify the effects of gas shale production and hydraulic fracturing on human health within the state of Pennsylvania, USA. These data will be linked to de-identified EHR data from GHS and the MyCode biobank of genetic data. Another expanding area of collected exposure data is through human biomonitoring of exposures, where the concentration of natural and synthetic compounds circulating in the body, such as in the blood or tissues, are evaluated and used as environmental exposure data. These additional resources of environmental data will be increasingly valuable in the exploration of $\mathrm{G} \times \mathrm{E}$.

With increasing collections of comprehensive environmental exposure data, such as within the field autism spectrum disorder research [156] and through the use of consensus standardized measures for Phenotypes and Exposures (PhenX) [7], comprehensive tests for $\mathrm{G} \times \mathrm{E}$ can be explored. However, it is important to note that exhaustive $\mathrm{G} \times \mathrm{E}$ evaluation can be computationally intensive and incur a high multiple testing burden. Environment-wide association studies are one way to identify key environmental exposures that can be selected for followup in subsequent $\mathrm{G} \times \mathrm{E}$ studies. Environment-wide association studies explore the association between a comprehensive range of environmental exposures and disease outcome (much like PheWAS), identifying key environmental exposures for further study. This method has been demonstrated using laboratory [157-160] and survey [12] measures. Dietary-wide association studies are another example exploring the association between dietary exposures and phenotypes in a high-throughput fashion, again identifying key dietary exposures for further research in $G \times E$ contexts [161], as well as being able to incorporate potential environmental exposures into high-throughput PheWAS analyses.

\section{Conclusions}

Embracing the inherent complexity of biological data to uncover the etiology of disease and drug response is a key challenge and opportunity for the future of genetic epidemiology. Much of the focus in genetic epidemiology and pharmacogenomics has been on the association between single genetic variants and a single disease at a time. However, to elucidate more of the impact of genetic architecture on these complex traits will require the incorporation of more of the underlying complexity into the analyses. As detailed with examples in this paper, in the future we can leverage even more of this complexity in novel ways to highlight critical contributors to diseases and drug response. Methodologies that show promise for leveraging this complexity include using phenomics approaches, more -omic data, PheWAS, and environment incorporated into analyses for a more complete, holistic view of the factors that impact the human phenome. 


\section{References}

$>1$ Lieberman-Aiden E, et al: Comprehensive mapping of long-range interactions reveals folding principles of the human genome. Science 2009;326;289-293.

$>2$ Kellis M, et al: Defining functional DNA elements in the human genome. Proc Natl Acad Sci USA 2014;111:6131-6138.

3 Choudhry S, et al: Dissecting complex diseases in complex populations: asthma in Latino Americans. Proc Am Thorac Soc 2007;4:226233.

74 Maher B: Personal genomes: the case of the missing heritability. Nature 2008;456:18-21.

5 Tyler AL, Asselbergs FW, Williams SM, Moore JH: Shadows of complexity: what biological networks reveal about epistasis and pleiotropy. Bioessays 2009;31:220-227.

-6 Ritchie MD, Holzinger ER, Li R, Pendergrass SA, Kim D: Methods of integrating data to uncover genotype-phenotype interactions. Nat Rev Genet 2015;16:85-97.

7 McCarty CA, et al: Validation of PhenX measures in the personalized medicine research project for use in gene/environment studies. BMC Med Genomics 2014;7:3

$>8$ Hamilton CM, et al: The PhenX Toolkit: get the most from your measures. Am J Epidemiol 2011;174:253-260.

$>9$ McCarty CA, et al: PhenX RISING: real world implementation and sharing of PhenX measures. BMC Med Genomics 2014;7:16.

10 Pan H, et al: Using PhenX measures to identify opportunities for cross-study analysis. Hum Mutat 2012;33:849-857.

11 Pendergrass SA, et al: Next-generation analysis of cataracts: determining knowledge driven gene-gene interactions using Biofilter, and gene-environment interactions using the PhenX Toolkit. Pac Symp Biocomput 2013; 147-158.

-12 Hall MA, et al: Environment-wide association study (EWAS) for type 2 diabetes in the Marshfield Personalized Medicine Research Project Biobank. Pac Symp Biocomput 2014;200-211.

13 Ellis K, et al: Identifying Active Travel Behaviors in Challenging Environments Using GPS, Accelerometers, and Machine Learning Algorithms. Front Public Health 2014;2:36.

$\checkmark 14$ McCrorie PR, Fenton C, Ellaway A: Combining GPS, GIS, and accelerometry to explore the physical activity and environment relationship in children and young people - a review. Int J Behav Nutr Phys Act 2014;11:93.

$>15$ Burgoine T, Jones AP, Brouwer RJN, Neelon SEB: Associations between BMI and home, school and route environmental exposures estimated using GPS and GIS: do we see evidence of selective daily mobility bias in children? Int J Health Geogr 2015;14:8.

-16 Lanktree MB, Hassell RG, Lahiry P, Hegele RA: Phenomics: expanding the role of clinical evaluation in genomic studies. J Investig Med 2010;58:700-706.
17 Oti M, Huynen MA, Brunner HG: Phenome connections. Trends Genet 2008;24:103-106.

18 Houle D, Govindaraju DR, Omholt S: Phenomics: the next challenge. Nat Rev Genet 2010;11:855-866.

19 Viti C, Decorosi F, Marchi E, Galardini M, Giovannetti L: High-throughput phenomics. Methods Mol Biol 2015;1231:99-123.

20 Ritchie MD, et al: Robust replication of genotype-phenotype associations across multiple diseases in an electronic medical record. Am J Hum Genet 2010;86:560-572.

21 Crawford DC, et al: eMERGEing progress in genomics-the first seven years. Front Genet 2014;5:184.

22 Gottesman O, et al: The Electronic Medical Records and Genomics (eMERGE) Network: past, present, and future. Genet Med 2013;15: 761-771.

23 McCarty CA, et al: The eMERGE Network: a consortium of biorepositories linked to electronic medical records data for conducting genomic studies. BMC Med Genomics 2011;4: 13.

24 Hays J, et al: The Women's Health Initiative recruitment methods and results. Ann Epidemiol 2003;13:S18-S77.

25 Friedman GD, et al: CARDIA: study design, recruitment, and some characteristics of the examined subjects. J Clin Epidemiol 1988;41: 1105-1116.

26 Design of the Women's Health Initiative clinical trial and observational study. The Women's Health Initiative Study Group. Control Clin Trials 1998;19:61-109.

27 Fried LP, et al: The Cardiovascular Health Study: design and rationale. Ann Epidemiol 1991;1:263-276.

28 Kolonel LN, Altshuler D, Henderson BE: The multiethnic cohort study: exploring genes, lifestyle and cancer risk. Nat Rev Cancer 2004; 4:519-527.

29 Bild DE, et al: Multi-ethnic study of atherosclerosis: objectives and design. Am J Epidemiol 2002;156:871-881.

30 Goodpaster BH, et al: Attenuation of skeletal muscle and strength in the elderly: the Health ABC Study. J Appl Physiol 2001;90:21572165 .

31 Signorello LB, Hargreaves MK, Blot WJ: The Southern Community Cohort Study: investigating health disparities. J Health Care Poor Underserved 2010;21:26-37.

32 Taylor HA: The Jackson Heart Study: an overview. Ethn Dis 2005;15:S6-1-S6-3.

33 Sorlie PD, et al: Design and implementation of the Hispanic Community Health Study/ Study of Latinos. Ann Epidemiol 2010;20: 629-641.

34 Hofman A, et al: The Rotterdam Study: 2014 objectives and design update. Eur J Epidemiol 2013;28:889-926.
35 Splansky GL, et al: The Third Generation Cohort of the National Heart, Lung, and Blood Institute's Framingham Heart Study: design, recruitment, and initial examination. Am J Epidemiol 2007;165:1328-1335.

36 Tatonetti NP, Ye PP, Daneshjou R, Altman RB: Data-driven prediction of drug effects and interactions. Sci Transl Med 2012;4: $125 \mathrm{ra3} 1$.

37 Fitzmaurice GM, Laird NM, Ware JH: Applied Longitudinal Analysis. Hoboken, John Wiley \& Sons, 2012.

38 Sitlani CM, et al: Generalized estimating equations for genome-wide association studies using longitudinal phenotype data. Stat Med 2015;34:118-130.

39 Kerem B, Kerem E: The molecular basis for disease variability in cystic fibrosis. Eur J Hum Genet 1996;4:65-73.

40 Chandriani S, et al: A core MYC gene expression signature is prominent in basal-like breast cancer but only partially overlaps the core serum response. PLoS One 2009;4:e6693.

41 Pendergrass SA, et al: Limited systemic sclerosis patients with pulmonary arterial hypertension show biomarkers of inflammation and vascular injury. PLoS One 2010;5:e12106.

42 Pendergrass SA, et al: Intrinsic gene expression subsets of diffuse cutaneous systemic sclerosis are stable in serial skin biopsies. J Investig Dermatol 2012;132:1363-1373.

43 Milano A, et al: Molecular subsets in the gene expression signatures of scleroderma skin. PLoS One 2008;3:e2696.

44 Haldar P, et al: Cluster Analysis and Clinical Asthma Phenotypes. Am J Respir Crit Care Med 2008;178:218-224.

-45 Wardlaw AJ, Silverman M, Siva R, Pavord ID, Green R: Multi-dimensional phenotyping: towards a new taxonomy for airway disease. Clin Exp Allergy 2005;35:1254-1262.

46 Lahiry P, et al: A multiplex human syndrome implicates a key role for intestinal cell kinase in development of central nervous, skeletal, and endocrine systems. Am J Hum Genet 2009;84:134-147.

47 Aschard $\mathrm{H}$, et al: Maximizing the power of principal-component analysis of correlated phenotypes in genome-wide association studies. Am J Hum Genet 2014;94:662-676.

48 Van der Sluis S, Posthuma D, Dolan CV: TATES: Efficient Multivariate genotype-phenotype analysis for genome-wide association studies. PLoS Genet 2013;9:e1003235.

49 Kim S, Sohn K-A, Xing EP: A multivariate regression approach to association analysis of a quantitative trait network. Bioinformatics 2009;25:i204-i212.

50 Hwang T, et al: Co-clustering phenome - genome for phenotype classification and disease gene discovery. Nucleic Acids Res 2012; 40:e146.

-51 Dettling M, Bühlmann P: Supervised clustering of genes. Genome Biol 2002;3:research0069-research0069.15. 
52 D'haeseleer P: How does gene expression clustering work? Nat Biotech 2005;23:14991501.

-53 Burgel PR, Paillasseur JL, Roche N: Identification of clinical phenotypes using cluster analyses in COPD patients with multiple comorbidities. Bio Med Res Int 2014;2014:420134.

54 Roque FS, et al: Using electronic patient records to discover disease correlations and stratify patient cohorts. PLoS Comput Biol 2011;7:e1002141.

-55 Moore WC, et al: Sputum neutrophil counts are associated with more severe asthma phenotypes using cluster analysis. J Allergy Clin Immunol 2014;133:1557-1563.e5.

56 Kidd BA, Peters LA, Schadt EE, Dudley JT: Unifying immunology with informatics and multiscale biology. Nat Immunol 2014;15 118-127.

57 Goh K-I, et al: The human disease network. Proc Natl Acad Sci USA 2007; 104:8685-8690.

58 Goh K-I, Choi I-G: Exploring the human diseasome: the human disease network. Brief Funct Genomics 2012;11:533-542.

59 Darabos C, et al: The multiscale backbone of the human phenotype network based on biological pathways. BioData Min 2014;7:1.

60 Darabos C, Harmon SH, Moore JH: Using the bipartite human phenotype network to reveal pleiotropy and epistasis beyond the gene. Pac Symp Biocomput 2014;188-199.

61 Wagner GP, Zhang J: The pleiotropic structure of the genotype-phenotype map: the evolvability of complex organisms. Nat Rev Genet 2011;12:204-213.

-62 Solovieff N, Cotsapas C, Lee PH, Purcell SM, Smoller JW: Pleiotropy in complex traits: challenges and strategies. Nat Rev Genet 2013;14:483-495.

63 Tyler AL, Crawford DC, Pendergrass SA: The detection and characterization of pleiotropy: discovery, progress, and promise. Brief Bioinform, in press.

64 Paaby AB, Rockman MV: The many faces of pleiotropy. Trends Genet 2013;29:66-73.

-65 Corder EH, et al: The apolipoprotein E E4 allele and sex-specific risk of Alzheimer's disease. JAMA 1995;273:373-374.

66 Sing CF, Davignon J: Role of the apolipoprotein E polymorphism in determining normal plasma lipid and lipoprotein variation. Am J Hum Genet 1985;37:268-285.

-67 Zhernakova A, van Diemen CC, Wijmenga C: Detecting shared pathogenesis from the shared genetics of immune-related diseases. Nat Rev Genet 2009; 10:43-55.

68 Huang W, Wang P, Liu Z, Zhang L: Identifying disease associations via genome-wide association studies. BMC Bioinformatics 2009; 10(suppl 1):S68.

-69 Lees CW, Barrett JC, Parkes M, Satsangi J: New IBD genetics: common pathways with other diseases. Gut 2011;60:1739-1753.

70 Pierce BL, Ahsan H: Genome-wide 'pleiotropy scan' identifies HNF1A region as a novel pancreatic cancer susceptibility locus. Cancer Res 2011;71:4352-4358.
1 Voight BF, et al: Twelve type 2 diabetes susceptibility loci identified through large-scale association analysis. Nat Genet 2010;42:579589.

72 Huang J, Johnson AD, O’Donnell CJ: PRIMe: a method for characterization and evaluation of pleiotropic regions from multiple genomewide association studies. Bioinformatics 2011;27:1201-1206.

73 Okada Y, et al: Genome-wide association study for C-reactive protein levels identified pleiotropic associations in the IL6 locus. Hum Mol Genet 2011;20:1224-1231.

74 Hindorff LA, et al: Potential etiologic and functional implications of genome-wide association loci for human diseases and traits. Proc Natl Acad Sci USA 2009;106:9362-9367.

75 Sivakumaran S, et al: Abundant pleiotropy in human complex diseases and traits. Am J Hum Genet 2011;89:607-618.

76 Romeo G, McKusick VA: Phenotypic diversity, allelic series and modifier genes. Nat Genet 1994;7:451-453.

77 Gamazon ER, et al: SCAN: SNP and copy number annotation. Bioinformatics 2010;26: 259-262.

78 Moore CB, Wallace JR, Frase AT, Pendergrass SA, Ritchie MD: BioBin: a bioinformatics tool for automating the binning of rare variants using publicly available biological knowledge. BMC Med Genomics 2013;6(suppl 2):S6.

79 Ionita-Laza I, Lee S, Makarov V, Buxbaum JD, Lin X: Sequence kernel association tests for the combined effect of rare and common variants. Am J Hum Genet 2013;92:841-853.

$80 \mathrm{Wu}$ MC, et al: Rare-variant association testing for sequencing data with the sequence kernel association test. Am J Hum Genet 2011;89: 82-93.

81 Redon R, et al: Global variation in copy number in the human genome. Nature 2006;444: 444-454.

82 Coe BP, Girirajan S, Eichler EE: The genetic variability and commonality of neurodevelopmental disease. Am J Med Genet C Semin Med Genet 2012;160C:118-129.

83 Girirajan S, et al: Relative burden of large $\mathrm{CNVs}$ on a range of neurodevelopmental phenotypes. PLoS Genet 2011;7:e1002334.

84 Girirajan S, et al: Global increases in both common and rare copy number load associated with autism. Hum Mol Genet 2013;22: 2870-2880.

85 De Visser JAGM, Cooper TF, Elena SF: The causes of epistasis. Proc Biol Sci 2011;278: 3617-3624.

86 Turner SD, et al: Knowledge-driven multi-locus analysis reveals gene-gene interactions influencing HDL cholesterol level in two independent EMR-linked biobanks. PLoS One 2011;6:e19586.

87 Bush WS, et al: A knowledge-driven interaction analysis reveals potential neurodegenerative mechanism of multiple sclerosis susceptibility. Genes Immun 2011;12:335-340.
88 Pendergrass SA, et al: Genomic analyses with biofilter 2.0: knowledge driven filtering, annotation, and model development. BioData Mining 2013;6:25.

89 Bush WS, Dudek SM, Ritchie MD: Biofilter: a knowledge-integration system for the multi-locus analysis of genome-wide association studies. Pac Symp Biocomput 2009; 368-379.

$90 \mathrm{Hu} \mathrm{T}$, Darabos C, Cricco ME, Kong E, Moore JH: Genome-wide genetic interaction analysis of glaucoma using expert knowledge derived from human phenotype networks. Pac Symp Biocomput 2015;20: 207-218.

-91 Pan Q, Hu T, Moore JH: Epistasis, complexity, and multifactor dimensionality reduction. Methods Mol Biol 2013;1019:465-477.

92 Ritchie MD, et al: Multifactor-dimensionality reduction reveals high-order interactions among estrogen-metabolism genes in sporadic breast cancer. Am J Hum Genet 2001; 69:138-147.

93 Holzinger ER, Dudek SM, Frase AT, Pendergrass SA, Ritchie MD: ATHENA: the analysis tool for heritable and environmental network associations. Bioinformatics 2014; 30:698-705.

94 Holzinger ER, et al: ATHENA: a tool for meta-dimensional analysis applied to genotypes and gene expression data to predict HDL cholesterol levels. Pac Symp Biocomput 2013;385-396.

-95 Turner SD, Dudek SM, Ritchie MD: ATHENA: a knowledge-based hybrid backpropagation-grammatical evolution neural network algorithm for discovering epistasis among quantitative trait Loci. BioData Mining 2010;3:5.

-96 Beam AL, Motsinger-Reif A, Doyle J: Bayesian neural networks for detecting epistasis in genetic association studies. BMC Bioinformatics 2014; 15:368.

-97 Ritchie MD: Finding the epistasis needles in the genome-wide haystack. Methods Mol Biol 2015;1253:19-33.

98 Liu C, Ma J, Amos CI: Bayesian variable selection for hierarchical gene-environment and gene-gene interactions. Hum Genet 2015; 134:23-36.

99 Curk T, Rot G, Zupan B: SNPsyn: detection and exploration of SNP-SNP interactions. Nucleic Acids Res 2011;39:W444-W449.

100 Tyler AL, Lu W, Hendrick JJ, Philip VM, Carter GW: CAPE: an R package for combined analysis of pleiotropy and epistasis. PLoS Comput Biol 2013;9:e1003270.

101 Philip VM, Tyler AL, Carter GW: Dissection of complex gene expression using the combined analysis of pleiotropy and epistasis. Pac Symp Biocomput 2014;200-211.

102 Tyler AL, McGarr TC, Beyer BJ, Frankel WN, Carter GW: A genetic interaction network model of a complex neurological disease. Genes Brain Behav 2014;13:831-840. 
103 Holzinger ER, Ritchie MD: Integrating heterogeneous high-throughput data for metadimensional pharmacogenomics and disease-related studies. Pharmacogenomics 2012;13:213-222.

104 Pendergrass SA, et al: The use of phenomewide association studies (PheWAS) for exploration of novel genotype-phenotype relationships and pleiotropy discovery. Genet Epidemiol 2011;35:410-422.

105 Pendergrass SA, Ritchie MD: Phenomewide association studies: leveraging comprehensive phenotypic and genotypic data for discovery. Curr Genet Med Rep, in press.

106 Pendergrass SA, et al: Phenome-wide association study (PheWAS) for detection of pleiotropy within the Population Architecture using Genomics and Epidemiology (PAGE) Network. PLoS Genet 2013;9: e1003087.

107 Hall MA, et al: Detection of pleiotropy through a Phenome-wide association study (PheWAS) of epidemiologic data as part of the Environmental Architecture for Genes Linked to Environment (EAGLE) study. PLoS Genet 2014;10:e1004678.

108 Denny JC; et al: PheWAS: demonstrating the feasibility of a phenome-wide scan to discover gene-disease associations. Bioinformatics 2010;26:1205-1210.

109 Hebbring SJ, et al: A PheWAS approach in studying HLA-DRB1*1501. Genes Immun 2013;14:187-191.

110 Namjou B, et al: Phenome-wide association study (PheWAS) in EMR-linked pediatric cohorts, genetically links PLCL1 to speech language development and IL5-IL13 to eosinophilic esophagitis. Front Genet 2014;5: 401.

-111 Denny JC, et al: Systematic comparison of phenome-wide association study of electronic medical record data and genomewide association study data. Nat Biotechnol 2013;31:1102-1110.

112 Verma A, et al: Exploring the relationship between immune system related genetic variants and complex traits and disease through a phenome-wide association study (PheWAS). Submitted.

-113 Moore CB, et al: Phenome-wide association study relating pretreatment laboratory parameters with human genetic variants in AIDS clinical trials group protocols. Open Forum Infect Dis 2015;2:ofu113.

114 Pendergrass SA, et al: An investigation of pleiotropy using PheWAS in African Americans in the population architecture using genomics and epidemiology (PAGE) network. Submitted.

-115 Tryka KA, et al: NCBI's database of genotypes and phenotypes: dbGaP. Nucleic Acids Res 2014;42:D975-D979.

-116 Evangelou E, Ioannidis JPA: Meta-analysis methods for genome-wide association studies and beyond. Nat Rev Genet 2013;14:379389.
117 Moore CB, Wallace JR, Frase AT, Pendergrass SA, Ritchie MD: Using biobin to explore rare variant population stratification. Pac Symp Biocomput 2013;332-343.

118 Bansal V, Libiger O, Torkamani A, Schork NJ: Statistical analysis strategies for association studies involving rare variants. Nat Rev Genet 2010;11:773-785.

119 Mitchell SL, et al: Investigating the relationship between mitochondrial genetic variation and cardiovascular-related traits to develop a framework for mitochondrial phenome-wide association studies. BioData Min 2014;7:6.

120 Dumitrescu L, et al: Towards a phenomewide catalog of human clinical traits impacted by genetic ancestry. Submitted.

121 Neuraz A, et al: Phenome-wide association studies on a quantitative trait: application to TPMT enzyme activity and thiopurine therapy in pharmacogenomics. PLoS Comput Biol 2013;9:e1003405.

122 Kim S, Xing EP: Statistical estimation of correlated genome associations to a quantitative trait network. PLoS Genet 2009;5: e1000587.

123 O'Reilly PF, et al: MultiPhen: Joint model of multiple phenotypes can increase discovery in GWAS. PLoS One 2012;7:e34861.

124 Pendergrass SA, Dudek SM, Crawford DC, Ritchie MD: Synthesis-View: visualization and interpretation of SNP association results for multi-cohort, multi-phenotype data and meta-analysis. BioData Min 2010;3:10.

125 Pendergrass S, Dudek SM, Roden DM, Crawford DC, Ritchie MD: Visual integration of results from a large DNA biobank (BioVU) using synthesis-view. Pac Symp Biocomput 2011;265-275.

126 Pendergrass SA, Dudek SM, Crawford DC, Ritchie MD: Visually integrating and exploring high throughput Phenome-Wide Association Study (PheWAS) results using PheWAS-View. BioData Min 2012;5:5.

127 Wolfe D, Dudek S, Ritchie MD, Pendergrass SA: Visualizing genomic information across chromosomes with Pheno Gram. BioData Min 2013;6:18.

128 R Development Core Team: R: A Language and Environment for Statistical Computing. 2008. http://www.R-project.org.

129 Wickham H: ggplot2: elegant graphics for data analysis. New York, Springer.

130 Kanehisa M, Goto S: KEGG: kyoto encyclopedia of genes and genomes. Nucleic Acids Res 2000;28:27-30.

131 Cline MS, et al: Integration of biological networks and gene expression data using Cytoscape. Nat Protocols 2007;2:2366-2382.

132 Saito R, et al: A travel guide to Cytoscape plugins. Nat Methods 2012;9:1069-1076.

133 Wang K, Li M, Hakonarson H: Analysing biological pathways in genome-wide association studies. Nat Rev Genet 2010;11:843854 .
134 Yaspan BL, et al: Genetic analysis of biological pathway data through genomic randomization. Hum Genet 2011;129:563-571.

135 Williams SM, Haines JL: Correcting away the hidden heritability. Ann Hum Genet 2011;75:348-350.

136 Shelton JF, Tancredi DJ, Hertz-Picciotto I: Independent and dependent contributions of advanced maternal and paternal ages to autism risk. Autism Res 2010;3:30-39.

137 Durkin MS. et al: Advanced parental age and the risk of autism spectrum disorder. Am J Epidemiol 2008;168:1268-1276.

138 Cheslack-Postava K, Liu K, Bearman PS: Closely spaced pregnancies are associated with increased odds of autism in California sibling births. Pediatrics 2011;127:246-253.

139 Beard CM, Panser LA, Katusic SK: Is excess folic acid supplementation a risk factor for autism? Med Hypotheses 2011;77:15-17.

140 Schmidt RJ, et al: Prenatal vitamins, onecarbon metabolism gene variants, and risk for autism. Epidemiology 2011;22:476-485.

141 Croen LA, Grether JK, Yoshida CK, Odouli R, Hendrick V: Antidepressant use during pregnancy and childhood autism spectrum disorders. Arch Gen Psychiatry 2011;68: 1104-1112.

142 Gardener H, Spiegelman D, Buka SL: Perinatal and neonatal risk factors for autism: a comprehensive meta-analysis. Pediatrics 2011;128:344-355.

143 Hultman CM, Sandin S, Levine SZ, Lichtenstein P, Reichenberg A: Advancing paternal age and risk of autism: new evidence from a population-based study and a meta-analysis of epidemiological studies. Mol Psychiatry 2011;16:1203-1212.

144 Larsson M, Weiss B, Janson S, Sundell J, Bornehag C-G: Associations between indoor environmental factors and parentalreported autistic spectrum disorders in children 6-8 years of age. Neurotoxicology 2009;30:822-831.

145 Lyall K, Pauls DL, Santangelo S, Spiegelman D, Ascherio A: Maternal early life factors associated with hormone levels and the risk of having a child with an autism spectrum disorder in the nurses health study II. J Autism Dev Disord 2011;41:618-627.

146 Roberts EM, et al: Maternal residence near agricultural pesticide applications and autism spectrum disorders among children in the California Central Valley. Environ Health Perspect 2007;115:1482-1489.

147 Mitchell MM, et al: Levels of select PCB and PBDE congeners in human postmortem brain reveal possible environmental involvement in 15q11-q13 duplication autism spectrum disorder. Environ Mol Mutagen 2012;53:589-598.

148 Hertz-Picciotto I, et al: Polybrominated diphenyl ethers in relation to autism and developmental delay: a case-control study. Environ Health 2011;10:1. 
149 Volk HE, Hertz-Picciotto I, Delwiche L, Lurmann F, McConnell R: Residential proximity to freeways and autism in the CHARGE study. Environ Health Perspect 2011;119: 873-877.

150 Volk HE, Lurmann F, Penfold B, Hertz-Picciotto I, McConnell R: Traffic-related air pollution, particulate matter, and autism. JAMA Psychiatry 2013;70:71-77.

151 Becerra TA, Wilhelm M, Olsen J, Cockburn M, Ritz B: Ambient air pollution and autism in Los Angeles County, California. Environ Health Perspect 2013;121:380-386.

152 Windham GC, Zhang L, Gunier R, Croen LA, Grether JK: Autism spectrum disorders in relation to distribution of hazardous air pollutants in the San Francisco bay area. Environ Health Perspect 2006;114:1438-1444.
153 Kalkbrenner AE, et al: Perinatal exposure to hazardous air pollutants and autism spectrum disorders at age 8. Epidemiology 2010; 21:631-641.

154 Roberts AL, et al: Perinatal air pollutant exposures and autism spectrum disorder in the children of Nurses' Health Study II participants. Environ Health Perspect 2013;121: 978-984.

155 Marcellus Shale Research Initiative. http:// www.geisinger.org/100/pdf/Marcellus_ Shale_Research_Case_for_Support_2013. pdf.

156 Hertz-Picciotto I, et al: The CHARGE Study: an epidemiologic investigation of genetic and environmental factors contributing to autism. Environ Health Perspect 2006;114: 1119-1125.
157 Patel CJ, Bhattacharya J, Butte AJ: An environment-wide association study (EWAS) on type 2 diabetes mellitus. PLoS One 2010; 5:e10746.

158 Patel CJ, et al: Investigation of maternal environmental exposures in association with self-reported preterm birth. Reprod Toxicol 2013;45C:1-7.

159 Patel CJ, Cullen MR, Ioannidis JPA, Butte AJ: Systematic evaluation of environmental factors: persistent pollutants and nutrients correlated with serum lipid levels. Int J Epidemiol 2012;41:828-843.

160 Tzoulaki I, et al: A nutrient-wide association study on blood pressure. Circulation 2012; 126:2456-2464.

161 Davis MA, et al: A dietary-wide association study (DWAS) of environmental metal exposure in US children and adults. PLoS One 2014;9:e104768 